\title{
Progress in Research on Cytotoxic T Lymphocyte-Associated Antigen 4 (CTLA-4) and Bladder Cancer
}

\author{
Wei-Ming Fang ${ }^{1}$, Chun Chen ${ }^{2}$
}

Cite this article: Fang WM, Chen C: Progress in research on cytotoxic T lymphocyte-associated antigen 4 (CTLA-4) and bladder cancer. Ann Urol Oncol 2019; 2(2): 8-14. https://doi. org/10.32948/auo.2019.01.22

\begin{abstract}
Cytotoxic T-lymphocyte-associated protein-4 (CTLA-4) was first discovered in 1987 and confirmed to be a protein that is mainly expressed on the surface of activated lymphocytes. CTLA-4 is expressed on the surface of T cells and binds to B7 expressed on antigen presenting cells (APCs) to potentially play a role in inhibiting lymphocyte proliferation. Inhibitors of CTLA-4 were developed to promote the anti-tumor effects of $T$ cells and inhibit tumor growth. CTLA-4, as an immune checkpoint, has been realized as an important therapeutic target in bladder cancer. Two main CTLA-4 inhibitors are currently used: ipilimumab is a first-generation IgG1 monoclonal antibody that targets CTLA-4, and it is completely synthetic; tremelimumab, representing another class of CTLA-4 inhibitors, is a monoclonal antibody against CTLA-4 that acts similarly to ipilimumab and binds specifically to CTLA-4. The two types of CTLA-4 inhibitors were found to improve the treatment effect in patients with bladder cancer in comparison to conventional agents. To review this topic, we searched recently published related articles.
\end{abstract}

Key words CTLA-4, bladder cancer, immune checkpoint

\footnotetext{
1. Department of Surgery, Fujian Provincial Cancer Hospital, Fuzhou City, Fujian Province, P.R. China.

2. Department of Surgery, Sanming First People's Hospital, Sanming City, Fujian Province, P.R. China.

Correspondence: Wei-Ming Fang (Department of Surgery, Fujian Provincial Cancer Hospital, NO. 420 Fuma Road, Jing'an District, Fuzhou City, Fujian Province, P.R. China; Email: kszhucn@126.com).
} 


\section{Introduction}

Bladder cancer is a common malignant tumor of the urinary system. In recent years, the incidence of bladder cancer in China has gradually increased, seriously affecting people's life and health [1]. The incidence of bladder cancer has certain characteristics. The overall incidence is higher in men than in women, in the city than in rural areas, and increases with age. The incidence of malignant urinary tumors in China is highest in patients over 60 years old [2]. Among clinical bladder cancer patients, $70 \%$ have non-muscle invasive bladder cancer, which is characterized by a high recurrence rate and low mortality, and $30 \%$ have myometrial invasive bladder cancer, of which approximately half are potentially lethal [3]. For non-muscle invasive bladder cancer, bladder infusion chemotherapy has achieved good therapeutic effects and has been classified as a routine treatment; however, no breakthrough shave been achieved for myometrial invasive bladder cancer [4]. As an emerging treatment for bladder cancer, immunotherapy has gradually attracted increasing attention [5-7].

\section{Anti-tumor effect of the immune system}

In recent years, people's understanding of the anti-tumor effect of the immune system has gradually improved: after identifying tumor-associated antigens, the human body can induce the activation, proliferation, and migration of effector cells such as cytotoxic T lymphocytes (CTL). Strangulation is performed to exert the anti-tumor effect of the immune system. In contrast, tumor cells have evolved multiple mechanisms to reduce this killing effect of the immune system through immune escape $[8,9]$.

The activation of $\mathrm{T}$ cells is critical during the anti-tumor immune system effect. T cell activation is regulated by the first and second signals. The first signal is derived from the recognition of antigen by $\mathrm{T}$ cells. Tumor-specific antigens are recognized by the major histocompatibility complex (MHC) on the surface of antigen- presenting cells (APCs) and presented to T cell receptors (TCRs) on the $T$ cell surface, generating the first signal. Stimulation of the simple first signal is not sufficient to complete the activation of $\mathrm{T}$ cells, and the participation of the second signal is also required. The second signal is produced by the interaction of the costimulatory molecule CD28 on the surface of T cells with the B7 ligand on the surface of antigen-presenting cells (APCs). After activation of the $\mathrm{T}$ cells, synergistic inhibitory molecules are expressed. The synergistic inhibitory molecules and synergistic promoting molecules compete with each other. By combining with ligands, respectively, stimulatory and inhibitory signals are generated. The two signals are in a state of subtle dynamic equilibrium and are activated. The immune system completes the anti-tumor effect, but it does not over-activate it, reducing the immune response to normal tissue injury (Figure 1) $[9,10]$. In this dynamic regulatory process, cytotoxic T-lymphocyte-associated protein-4 (CTLA-4) is an important synergistic inhibitory molecule. Tumor cells inhibit $\mathrm{T}$ cell activation via promoting the expression of CTLA-4 for immune escape, thus evading killing by the immune system. This mechanism also makes CTLA-4 an important target for anti-tumor immunotherapy drugs [11].

\section{Mechanism of action of CTLA-4}

CTLA-4 was first discovered in 1987 and confirmed to be a protein that is primarily expressed on the surface of activated lymphocytes. Experiments have shown that the absence of CTLA-4 in mice leads to extensive lymphoid tissue hyperplasia, suggesting that CTLA-4 may play a role in inhibiting lymphocyte proliferation [12]. With the development of molecular biology, people have acquired an improved understanding of the structure of CTLA-4: CTLA4 is a transmembrane protein that belongs to the immunoglobulin superfamily composed of three parts: the extracellular region, transmembrane region and intracellular region. The extracellular domain functions as a receptor for the $\mathrm{B} 7$ molecule, which is

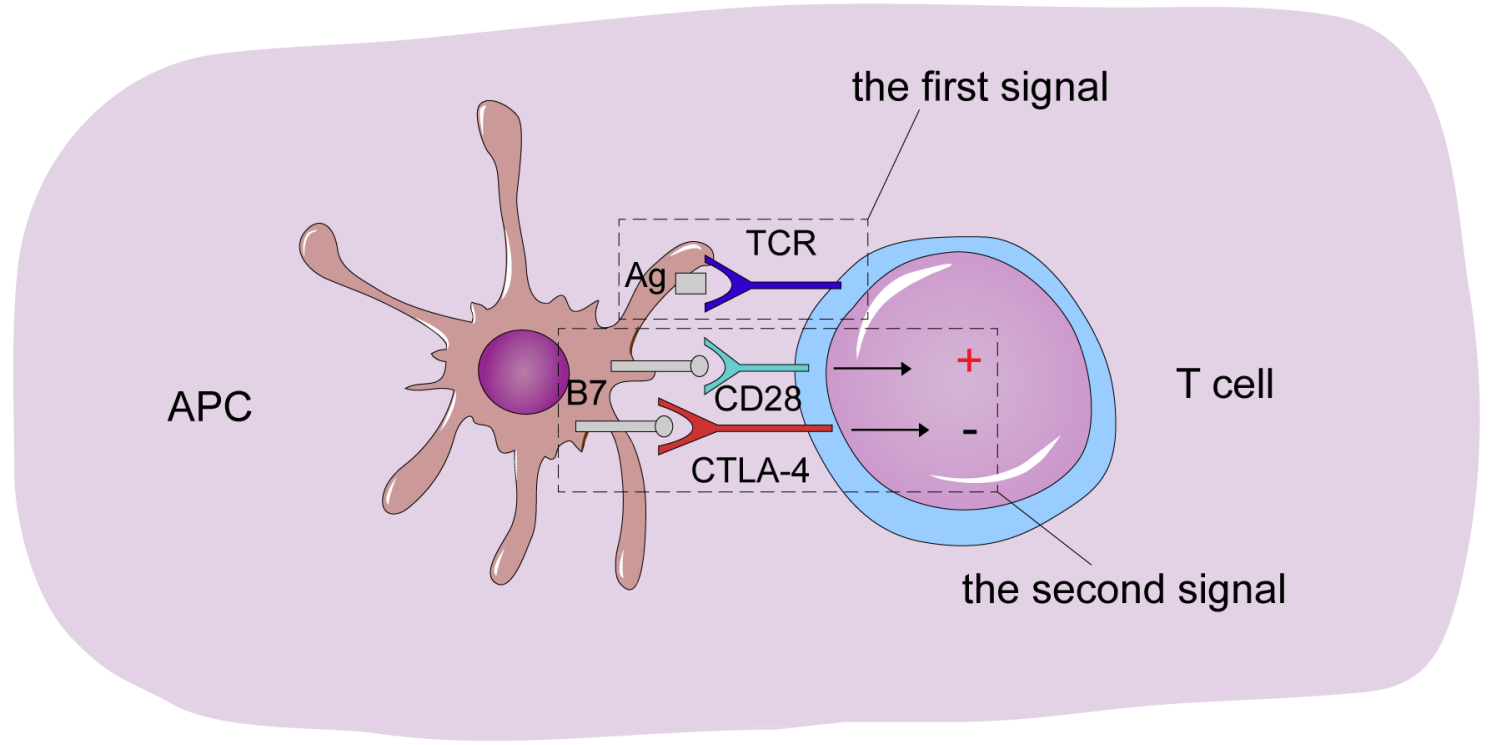

Figure 1: Introduction of three signals in $\mathrm{T}$ cells to activate antigen-presenting cells (APCs), which present tumor-related antigen to $\mathrm{T}$ cells that is bound by $\mathrm{T}$ cell receptors (TCRs). Another two signals, the combination of B7 ligand (expressed in APCs) and CD28 (expressed in T cells) and B7 ligand and CTLA-4 binding induce $T$ cell activation and inhibition, respectively. They are in a state of subtle dynamic equilibrium and are activated. The immune system completes the anti-tumor effect, but it does not over-activate it, reducing the immune response to normal tissue injury. CTLA-4, cytotoxic T-lymphocyte-associated protein-4; APCs, antigen-presenting cells; TCRs, T cell receptors; +, T cell activation; -, suppression of $T$ cell activation. 
composed of 116 amino acids; the intracellular region is composed of 34 amino acids, which constitutes the immune receptor tyrosine inhibitory motif (ITIM). The structure of the CD28 molecule has been determined, and its intracellular division is divided by an immunoreceptive activating motif (ITAM). The relative role of ITIM and ITAM constitutes the molecular biological basis for the opposite regulation of CTLA-4 and CD28 (Figure 1) [13].

The mechanism by which CTLA-4 inhibits $\mathrm{T}$ cell activation has not been fully elucidated. The current research results mainly focus on the binding of CTLA-4 and B7 ligand or CD28 and B7 ligand: CTLA-4 and CD28 molecules on the surface of T can combine with APC. The surface binding of B7 ligand and CTLA-4 is stronger than that of CD28 and B7 ligand. The binding of CD28 to $\mathrm{B} 7$ ligand produces a costimulatory signal that stimulates $\mathrm{T}$ cell activation, whereas the binding of CTLA-4 to B7 ligand produces a synergistic inhibitory signal that inhibits $\mathrm{T}$ cell activation $[8,9$, 11]. In addition, studies have shown that CTLA-4 may also downregulate tumor immune responses through various other pathways, such as inhibition of the cell cycle progression, interference with TCR signaling, and inhibition of apoptosis. Even if the specific mechanism of action of CTLA-4 is not fully understood, it does not affect the anti-tumor application of CTLA-4 inhibitors. Some CTLA-4 inhibitors have been approved by the Food and Drug Administration (FDA) for clinical treatment and have achieved positive results [14].

\section{Immunotherapy of CTLA-4 inhibitors and bladder tumors}

During construction of the mouse model in 1994, anti-CTLA-4 antibody was used to inhibit tumor growth, thereby revealing the anti-tumor effects of CTLA-4 immunological checkpoint inhibitors (mainly anti-CTLA-4 antibodies) [15]. Studies have shown that CTLA-4 inhibitors can efficiently bind to CTLA-4 molecules, reducing the number of CTLA-4 molecules that bind to $\mathrm{B} 7$ molecules and enhancing $\mathrm{T}$ cell activation by reducing the production of negative regulatory signals that inhibit $T$ cell activation. With the growth and infiltration of tumor- specific
$\mathrm{T}$ cells in the tumor microenvironment, the anti-tumor effect of the immune system is achieved. In addition, studies have shown that CTLA-4 inhibitors can reduce the number of regulatory $\mathrm{T}$ cells (Tregs) by interacting with $\mathrm{Fc} \gamma$ receptors on the surface of macrophages, thereby enhancing the ability of the immune system to kill tumor cells (Figure 2) [16, 17].

Two main CTLA-4 inhibitors are currently used. Ipilimumab is a first-generation IgG1 monoclonal antibody targeting CTLA-4, which is completely synthetic. Ipilimumab was the first CTLA4 inhibitory drug approved by the US FDA for the clinical treatment of unresectable or metastatic melanoma [18], and it can significantly prolong the median survival of patients [19], increasing the median overall survival (OS) of patients with metastatic melanoma to 10 months [20]. Based on numerous successful preclinical trials, ipilimumab has also been used in clinical research for bladder cancer in recent years. A phase II multicenter clinical study of metastatic urothelial carcinoma showed that the addition of ipilimumab increased the patient's response rate by $22 \%$ compared with conventional gemcitabine plus platinum-based chemotherapy. The overall response rate (ORR) was $69 \%$, and the 1-year OS was 61\% [21]. In addition, a clinical study confirmed that preoperative use of ipilimumab can significantly improve postoperative survival [22].

Tremelimumab is in another class of CTLA- 4 inhibitors; it is a monoclonal antibody against CTLA-4 that acts similarly to ipilimumab and specifically binds to CTLA-4. Clinical trials have confirmed that tremelimumab has a certain therapeutic effect on melanoma. In addition, it was approved for the treatment of malignant mesothelioma in 2015, and approximately $52 \%$ of patients were effectively controlled, with a median survival of 10.9 months [23]. However, the therapeutic effect of tremelimumab on bladder tumors remains to be discussed. The benefits and risks of using tremelimumab for bladder cancer must be confirmed in further clinical trials. Multi-drug combination is a research hotspot and current treatment trend. A series of clinical trials combining CTLA-4 inhibitors with other immune checkpoint inhibitors and CTLA-4 inhibitors combined with traditional chemotherapy drugs

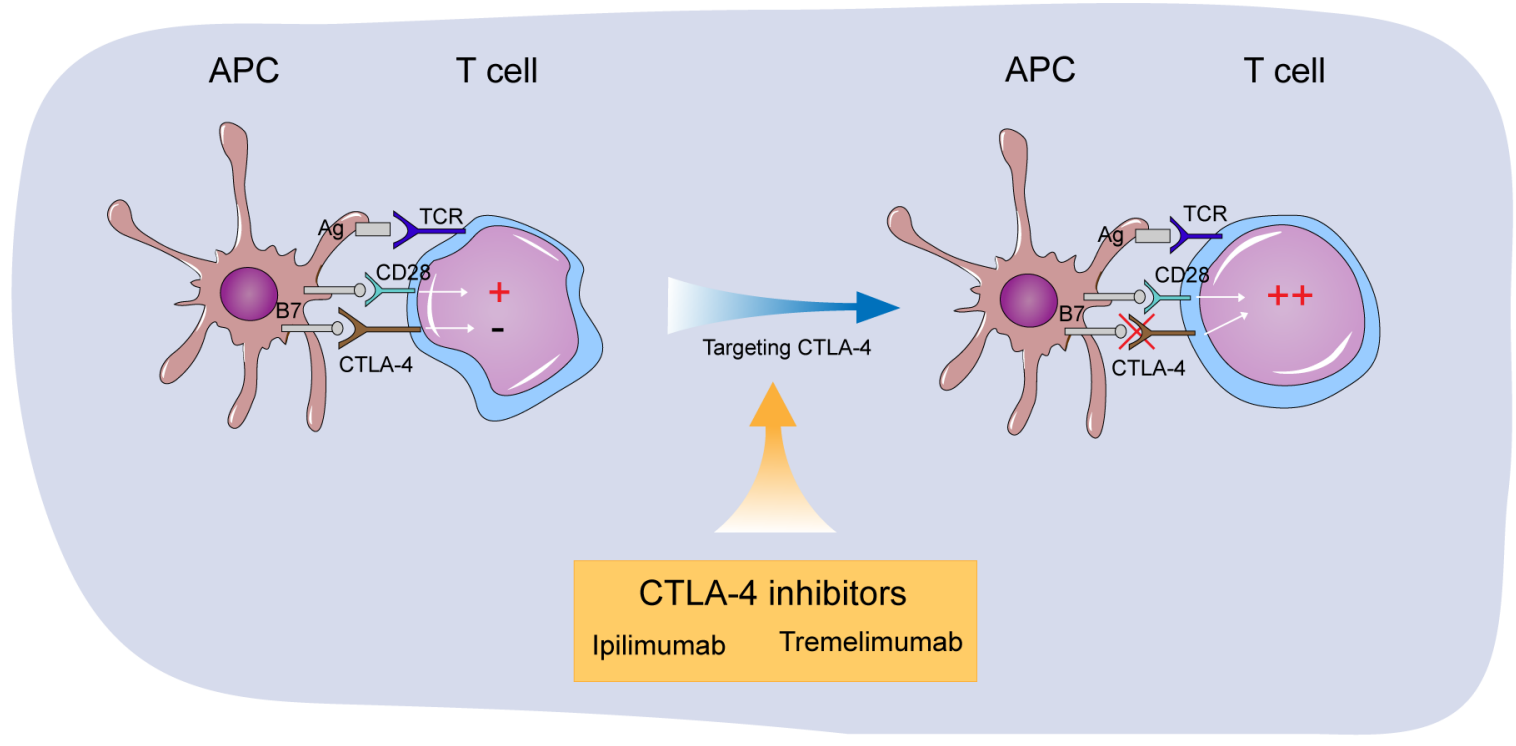

Figure 2: Mechanism of the two main CTLA-4 inhibitors currently used for the treatment of bladder cancer: ipilimumab and tremelimumab. CTL-A inhibitors, including ipilimumab and tremelimumab, play a role in inhibiting the function of CTLA-4, promoting T cell activities. CTLA-4, cytotoxic T-lymphocyte-associated protein-4; APCs, antigen presenting cells; TCRs, T cell receptors; +, T cell activation; -, suppression of $T$ cell activation. 


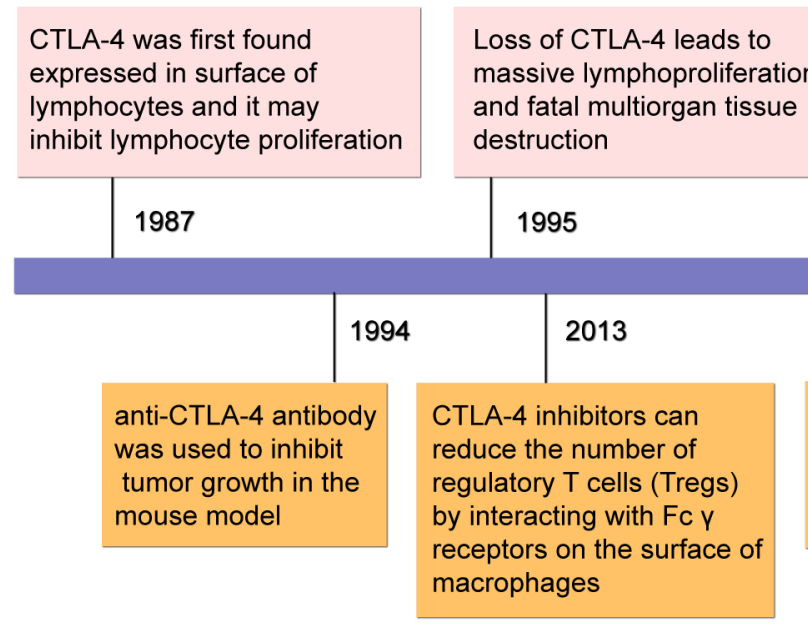

2014

Ipilimumab is the first generation of $\lg \mathrm{G} 1$ monoclonal antibody targeting CTLA-4
ITIM and ITAM constitutes the molecular biological basis for the opposite regulation of CTLA-4 and CD28

Figure 3: Timeline for the development of CTLA-4 and CTLA-4 inhibitors. CTLA-4, cytotoxic T-lymphocyte-associated protein-4; ITIM, immune receptor tyrosine inhibitory motif; ITAM, immunoreceptive activating motif.

are currently underway. At present, the available research results show that the compatibility of various drugs can significantly improve the response rate of bladder tumors to drugs, and the risk of using such drugs is simultaneously significantly improved. The selection of CTLA-4 inhibitors and the specification of doses to be used remain to be further addressed (Figure 3). Table 1 shows recent research results on CTLA-4 inhibitors for the treatment of bladder cancer [21, 24-26].

\section{Limitations of CTLA-4 inhibitors}

The emergence of immunological checkpoint inhibitors as a new adjuvant therapy provides novel ideas for the treatment of bladder cancer, resulting in a new treatment for patients with ineffective standard chemotherapy, such as the application of CTLA-4 inhibitors. There are some limitations to the application of CTLA4 inhibitors, encompassing mainly two aspects: first, CTLA4 inhibitors are ineffective in some patients; second, CTLA-4 inhibitors cause some adverse reactions [11].

In clinical applications, CTLA- 4 inhibitors are ineffective in patients with partial bladder tumors. Even if the dose is increased or combined with other immunomodulatory inhibitors, although the response rate of the drug can be improved, it is still ineffective for some patients. Thus, the construction of a suitable screening mechanism to rule out this population of patients who do not benefit from immunomodulatory point inhibitors is an urgent problem that must be solved.

In addition, studies have shown that immune checkpoint inhibitors also promote a series of adverse reactions while improving patient survival, the most prominent of which are immune-related adverse events (irAEs). irAEs not only affect the quality of life of patients, but they even force patients to stop treatment, and severe cases can lead to death [24]. Studies have shown that the use of immunosuppressants leads to the occurrence of varying degrees of irAEs, with a maximum incidence of $80.2 \%$, which can affect almost all organs and systems of the human body [27]. Common irAEs are described below.

1. Skin adverse reactions: Skin adverse reactions are the first adverse reactions to occur, starting at approximately 3 weeks. According to statistics, skin reactions such as redness, itching and vitiligo can affect $25.6 \% \sim 50.0 \%$ of patients and are often located on the limbs and trunk. The incidence of mossy skin reactions, mucosal lichen planus, and acute febrile neutrophilosis, among others, is generally low, usually affecting no more than $10 \%$ of patients. Although skin death is less common in patients with adverse reactions, due to its high incidence and subtle impact on patients' lives and psychology, early identification and intervention are still needed [28].

2. Digestive system adverse reactions: Digestive system adverse reactions are often manifested as diarrhea, colitis, abdominal pain and esophagitis. According to statistics, ipilimumab causes a higher incidence of diarrhea and colitis, reaching up to $32.8 \%$; the incidence of abdominal pain is lower at $5 \%$. In a Phase I clinical trial examining ipilimumab, 5 of 7 deaths were attributed to autoimmune enteritis [27]. Most enteritis-related irAEs are mild and often begin to appear 6 to 7 weeks after drug initiation. Clinical trials have shown that the incidence of enteritis-related irAEs increases with increasing doses of ipilimumab: in phase II clinical trials, the probability of an enteritis response in patients treated with $0.3,3$, and $10 \mathrm{mg} / \mathrm{kg}$ ipilimumab was $0 \%, 3 \%$ and $15 \%$, respectively [26]. It should be noted that patients with colitis are more likely to experience inflammatory reactions in the eye, especially uveitis. Therefore, patients with immune-associated colitis must also pay attention to the occurrence of ophthalmia [26]. 3. Hepatitis: Hepatitis caused by ipilimumab can occur at any time, usually within 8 to 12 weeks of drug initiation. Severe cases can lead to death. There are usually no clinical symptoms, with only the manifestation of changes in laboratory indicators, such as increases in aspartate aminotransferase, alanine aminotransferase and total bilirubin [30].

4. Endocrine system adverse reactions: Endocrine system adverse reaction shave an incidence of $10 \%$ [31]. The incidence of hyperthyroidism ranges from $0 \%-2.8 \%$, and the incidence of hypothyroidism from $0-9 \%$. The incidence of pituitary inflammation was found to be $1.8 \%$ and the incidence of adrenal insufficiency $1.5 \%$. Cases of autoimmune insulin-dependent diabetes mellitus caused by immunological checkpoint inhibitors have also been reported in the literature [32]. Studies have shown that the incidence of adverse reactions in the endocrine system is significantly positively correlated with the dose of CTLA-4.

In addition, immune-related adverse reactions can also affect the kidneys, eyes, lungs, and nervous system. 


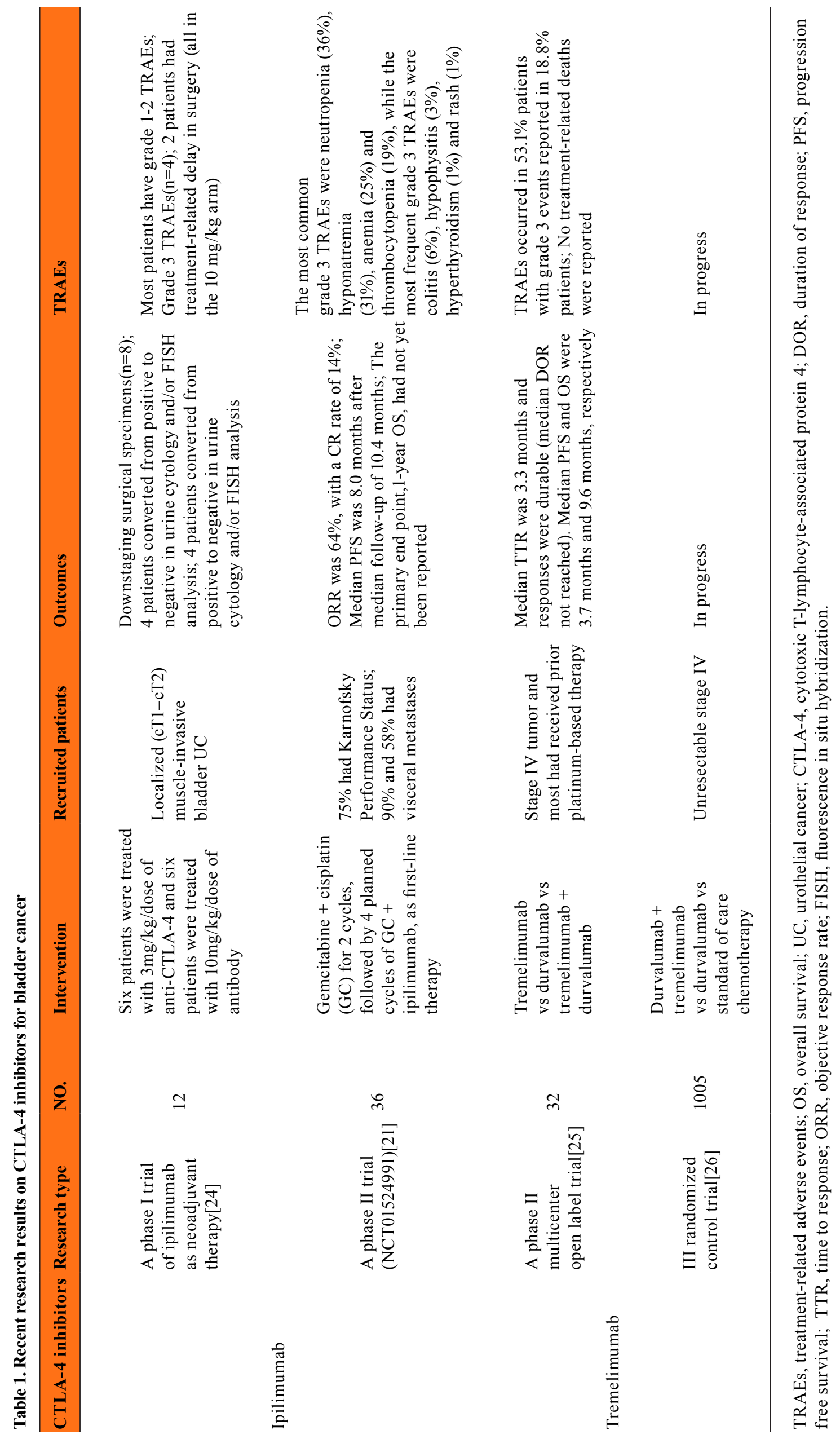


The etiology of irAEs is not clear and may be related to the following factors. 1. A part of the antigen is co-expressed on the surface of tumor and normal cells, and cross-presentation of this antigen part may impair the immune tolerance of normal patient tissues [33]. 2. In addition to activated T cells, CTLA-4 is also expressed on the surface of Treg cells, and CTLA-4 inhibitors cause extensive and non-specific activation of immune responses by limiting the function of Treg cells [34]. The diversity of 3T cells may also be involved in the progression of autoimmune inflammatory responses $[17,35]$. These mechanisms can cause the immune system to exert accidental injury to normal tissues while fighting tumors. Unfortunately, there are currently no mature and detailed identification procedures and treatment protocols for immune-related adverse reactions, thus hindering the application of CTLA-4 inhibitors to some extent.

\section{Application prospects of CTLA-4}

With the improvement of people's understanding of the internal relationship between the immune response and tumorigenesis, techniques to strengthen the tumor killing effect of the immune system and to reduce the immune escape of tumors have become a hot topic of current research. Immunoregulatory inhibitors have received widespread attention as a novel immune-related antitumor agent. Two main types of immunological checkpoint inhibitors are currently approved by the FDA: CTLA- 4 inhibitors (anti-CTLA-4 antibodies) and PD-1/PDL-1 inhibitors (antiPD1/PDL-1 antibodies). Although they are immunosuppressive molecules, their mechanism of action is different: CTLA-4 acts in the early stage of T cell activation; PD-1 acts in the late stage of T cell activation. Similar to the function of CTLA-4, PD-1 can also inhibit the activation and proliferation of $\mathrm{T}$ cells, but PD-I plays a role mainly after $\mathrm{T}$ cells migrate to the tumor microenvironment. Compared with CTLA-4, PD-1 has a stronger inhibitory effect; however, the role of CTLA-4 is not negligible [36, 37]. At present, we still do not fully understand the specific biological functions and molecular mechanism of CTLA-4. The role of CTLA4 in bladder tumorigenesis remains to be further understood. As research progresses, more CTLA-4-related drugs will be developed and applied in the clinic. Multi-drug combinations are a current a treatment trend, but the benefits and risks of multi-drug combination require further evaluation in clinical trials. Strategies to reduce or even eliminate the adverse reactions associated with immune checkpoint inhibitors, to screen the application of immune checkpoint inhibitors and other issues still need to be explored.

\section{Funding}

None.

\section{Ethics approval and consent to participate}

This study was not involving in any ethics events and human study.

\section{Contributions of each author}

WMF: Literature search and manuscript writting; CC: Manuscript writting.

\section{Competing interests}

The authors declare no conflict of interest with the work.

\section{References}

1. Pang C, Guan Y, Li H, Chen W, Zhu G: Urologic cancer in China. Jpn J Clin Oncol 2016, 46(6): 497-501.

2. He YT, Li DJ, Liang D, Zheng RS, Zhang SW, Zeng HM, Chen WQ, He J: [Incidence and mortality of bladder cancer in China, 2014]. Zhonghua Zhong Liu Za Zhi 2018, 40(9): 647-652.

3. Marcos-Gragera R, Mallone S, Kiemeney LA, Vilardell L, Malats N, Allory Y, Sant M, Group E-W: Urinary tract cancer survival in Europe 1999-2007: Results of the population-based study EUROCARE-5. Eur J Cancer 2015, 51(15): 2217-2230.

4. da Costa JB, Gibb EA, Nykopp TK, Mannas M, Wyatt AW, Black PC: Molecular tumor heterogeneity in muscle invasive bladder cancer: Biomarkers, subtypes, and implications for therapy. Urol Oncol 2018, https://doi.org/10.1016/j.urolonc.2018.11.015. Epub ahead of print.

5. Doyle E, Crew J, Mostafid H, Tuthill M, Cerundolo V, Gerristen W, Protheroe A: Urothelial cancer: a narrative review of the role of novel immuno-therapeutic agents with particular reference to the management of non- muscle invasive disease. BJU Int 2018, https:// doi.org/10.1111/bju.14643. Epub ahead of print.

6. Stuhler V, Maas JM, Bochem J, da Costa IA, Todenhofer T, Stenzl A, Bedke J: Molecular predictors of response to PD-1/PD-L1 inhibition in urothelial cancer. World J Urol 2018, https://oi.org/10.1007/ s00345-018-2538-6. Epub ahead of print.

7. Zibelman M, Ramamurthy C, Plimack ER: Emerging role of immunotherapy in urothelial carcinoma-Advanced disease. Urol Oncol 2016, 34(12): 538-547.

8. De Palma M: The role of the immune system in cancer: From mechanisms to clinical applications. Biochim Biophys Acta 2016, 1865(1): 1-2.

9. Pardoll DM: The blockade of immune checkpoints in cancer immunotherapy. Nat Rev Cancer 2012, 12(4): 252-264.

10. Thompson RH, Kwon ED, Allison JP: Inhibitors of B7-CD28 costimulation in urologic malignancies. Immunotherapy 2009, 1(1): 129-139.

11. Wakamatsu E, Mathis D, Benoist C: Convergent and divergent effects of costimulatory molecules in conventional and regulatory CD4+ T cells. Proc Natl Acad Sci U S A 2013, 110(3): 1023-1028.

12. Brunet JF, Denizot F, Luciani MF, Roux-Dosseto M, Suzan M, Mattei MG, Golstein P: A new member of the immunoglobulin superfamily--CTLA-4. Nature 1987, 328(6127): 267-270.

13. Walker LS, Sansom DM: Confusing signals: recent progress in CTLA-4 biology. Trends Immunol 2015, 36(2): 63-70.

14. Tai X, Van Laethem F, Pobezinsky L, Guinter T, Sharrow SO, Adams A, Granger L, Kruhlak M, Lindsten T, Thompson CB et al: Basis of CTLA-4 function in regulatory and conventional CD4(+) T cells. Blood 2012, 119(22): 5155-5163.

15. Leach DR, Krummel MF, Allison JP: Enhancement of antitumor immunity by CTLA-4 blockade. Science 1996, 271(5256): 17341736.

16. Bulliard Y, Jolicoeur R, Windman M, Rue SM, Ettenberg S, Knee DA, Wilson NS, Dranoff G, Brogdon JL: Activating Fc gamma receptors contribute to the antitumor activities of immunoregulatory receptor-targeting antibodies. J Exp Med 2013, 210(9): 1685-1693.

17. Simpson TR, Li F, Montalvo-Ortiz W, Sepulveda MA, Bergerhoff K, Arce F, Roddie C, Henry JY, Yagita H, Wolchok JD et al: Fcdependent depletion of tumor-infiltrating regulatory $\mathrm{T}$ cells codefines the efficacy of anti-CTLA-4 therapy against melanoma. $\mathrm{J}$ Exp Med 2013, 210(9): 1695-1710.

18. Boutros C, Tarhini A, Routier E, Lambotte O, Ladurie FL, Carbonnel F, Izzeddine H, Marabelle A, Champiat S, Berdelou A et al: Safety profiles of anti-CTLA-4 and anti-PD-1 antibodies alone and in combination. Nat Rev Clin Oncol 2016, 13(8): 473-486.

19. Eigentler TK, Schlaak M, Hassel JC, Loquai C, Stoffels I, Gutzmer R, Patzold S, Mohr P, Keller U, Starz H et al: Effectiveness and tolerability of ipilimumab: experiences from 198 patients included in a named-patient program in various daily-practice settings and 
multiple institutions. J Immunother 2014, 37(7): 374-381.

20. Ferrucci PF, Ascierto PA, Pigozzo J, Del Vecchio M, Maio M, Antonini Cappellini GC, Guidoboni M, Queirolo P, Savoia P, Mandala $\mathrm{M}$ et al: Baseline neutrophils and derived neutrophil-tolymphocyte ratio: prognostic relevance in metastatic melanoma patients receiving ipilimumab. Ann Oncol 2016, 27(4): 732-738.

21. Galsky MD, Wang H, Hahn NM, Twardowski P, Pal SK, Albany C, Fleming MT, Starodub A, Hauke RJ, Yu M et al: Phase 2 Trial of Gemcitabine, Cisplatin, plus Ipilimumab in Patients with Metastatic Urothelial Cancer and Impact of DNA Damage Response Gene Mutations on Outcomes. Eur Urol 2018, 73(5): 751-1859.

22. Camacho LH: CTLA-4 blockade with ipilimumab: biology, safety, efficacy, and future considerations. Cancer Med 2015, 4(5): 661-672.

23. Guazzelli A, Bakker E, Krstic-Demonacos M, Lisanti MP, Sotgia F, Mutti L: Anti-CTLA-4 therapy for malignant mesothelioma. Immunotherapy 2017, 9(3): 273-280.

24. Carthon BC, Wolchok JD, Yuan J, Kamat A, Ng Tang DS, Sun J, Ku G, Troncoso P, Logothetis CJ, Allison JP et al: Preoperative CTLA4 blockade: tolerability and immune monitoring in the setting of a presurgical clinical trial. Clin Cancer Res 2010, 16(10): 2861-2871.

25. Sharma P, Hyuk Sohn J, Joon Shin S, Oh DY, Keam B, Lee HJ, Canon JL, Kalinka-Warzocha E, Langenberg M, Allison J et al: Efficacy and tolerability of tremelimumab in locally advanced or metastatic urothelial carcinoma; 2017.

26. AstraZeneca: Study of MEDI4736 with or without tremelimumab versus standard of care chemotherapy in urothelial cancer. NLM identifier: NCT02516241 2017: Accessed September 13.

27. Hodi FS, O'Day SJ, McDermott DF, Weber RW, Sosman JA, Haanen JB, Gonzalez R, Robert C, Schadendorf D, Hassel JC et al: Improved survival with ipilimumab in patients with metastatic melanoma. $\mathrm{N}$ Engl J Med 2010, 363(8): 711-1823.

28. Rotte A, Bhandaru M, Zhou Y, McElwee KJ: Immunotherapy of melanoma: present options and future promises. Cancer Metastasis Rev 2015, 34(1): 115-128.

29. Koo PJ, Klingensmith WC, Lewis KD, Bagrosky BM, Gonzalez R: Anti-CTLA4 antibody therapy related complications on FDG PET/ CT. Clin Nucl Med 2014, 39(1): e93-96.

30. Bryce J, Boers-Doets CB: Non-rash dermatologic adverse events related to targeted therapies. Semin Oncol Nurs 2014, 30(3): 155-168.

31. Ryder M, Callahan M, Postow MA, Wolchok J, Fagin JA: Endocrinerelated adverse events following ipilimumab in patients with advanced melanoma: a comprehensive retrospective review from a single institution. Endocr Relat Cancer 2014, 21(2): 371-381.

32. Torino F, Corsello SM, Salvatori R: Endocrinological side-effects of immune checkpoint inhibitors. Curr Opin Oncol 2016, 28(4): 278287.

33. Hamanishi J, Mandai M, Konishi I: Immune checkpoint inhibition in ovarian cancer. Int Immunol 2016, 28(7): 339-348.

34. Littman DR: Releasing the Brakes on Cancer Immunotherapy. Cell 2015, 162(6): 1186-1190.

35. Zohar Y, Wildbaum G, Novak R, Salzman AL, Thelen M, Alon R, Barsheshet Y, Karp CL, Karin N: CXCL11-dependent induction of FOXP3-negative regulatory $\mathrm{T}$ cells suppresses autoimmune encephalomyelitis. J Clin Invest 2014, 124(5): 2009-2022.

36. Fahmy O, Khairul-Asri MG, Stenzl A, Gakis G: The current status of checkpoint inhibitors in metastatic bladder cancer. Clin Exp Metastasis 2016, 33(7): 629-635.

37. Petrylak DP: Immunotherapy: The Wave of the Future in Bladder Cancer? Clin Genitourin Cancer 2017, 15(3S): S3-S17. 